\title{
Flow of polymolecular films
}

\author{
N.V. Churaev \\ Institute of Physical Chemistry, Moscow, 117915, USSR
}

\begin{abstract}
Experimental investigation of film flow over the molecularly smooth surfaces of quartz capillaries has shown that the films of nonpolar liquids having the thickness $h \geqslant 1 \mathrm{~nm}$ flow as viscous bulk liquids. This is also the case for thin water films, but the corresponding calculations are complicated due to the nonuniformity in the film thickness on the partially wetted quartz surface.
\end{abstract}

Introduction The flow of polymolecular films, depending on their thickness, may be considered as viscous flow, or as two-dimensional surface diffusion. In the first case, equations of hydrodynamics must be used for describing the flow of films; and in the second case, the equations of diffusion. It is important to establish the scope of application of those approaches.

In this connection, here will be considered the experimental results relating to the flow of polymolecular liquid films over the molecular-smooth aurface of fine cylindrical capillariea (having the radius on the order of 0.1 to $1 \mathrm{\mu m}$ ) drawn from pure quartz (the content of $\mathrm{SIO}_{2}>99.99 \%$ ).

Film flow under the effect of pressure gradient The first technique consisted

in measuring the evaporation rates of liquids from the capillaries. For this purpose the rate of the meniscus retreat was measured with means of a microscope. In the case of low volatile liquids a considerable contribution to the evaporation rate is given by the film flow over the wall of the capillary.

In Fig. 1 are represented the results of such experiments on decane during its evaporation at low Knudson numbers $\lambda / r(\lambda)$ is the free path) into an evacuated chamber, where a definite relative pressure of vapour $\mathrm{p}_{0} / \mathrm{p}$ was maintained [1]. Measurements were carried out for the cap1llaries having the radii $r$ from 0.18 to $1.5 \mu \mathrm{m}$. Here $K$ is the permeability coefficient, included in the mass flow equation $q=K\left(p_{m}-p_{0}\right) / 1$, $\mathrm{g} / \mathrm{cm}^{2}$ sec, where $p_{m} \simeq p_{s}$ is the pressure of vapour over the meniscus, and 1 Is the distance from the mouth to the meniscus in a capillary. The values of $K$ are related to the coefficient of Knudsen diffusion of vapour $K_{1}=(8 r / 3)(\mu / 2 \pi R T)^{1 / 2}$ at $r / \lambda \rightarrow 0$, where $\mu$ is the mass of a mole,

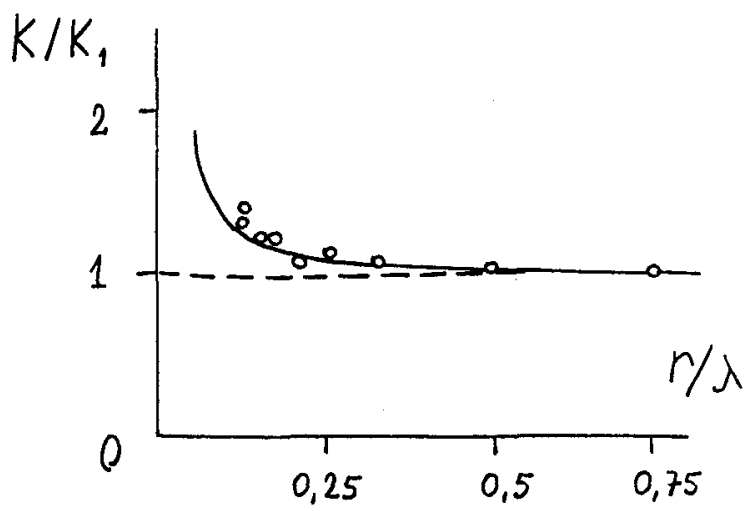

Fig. 1. The influence of the flow of polymolecular films on evaporation of decane from fine capillaries. 
$R$ is the gas constant, and $T$ is temperature. The dashed line indicates the theoretical dependence of $K_{1}(r / \lambda)$ for vapour flow. The experimental points for fine capillaries are located above this curve, and this is sgsociated with an additional mass transfer in a polymolecular film of decane.

The experimental data relating to the film thicknesses of about 5 to $1 \mathrm{~nm}$, can be described on the basis of the equation of hydrodynamics of liquid films, $q=\left(\rho h^{2} / 3 \eta\right) \nabla \Pi$, where $\Pi(h)$ is the disjoining pressure [2], $\rho$ is the density, and $\mu$ is the viscosity of liquid in the film. Using the condition of the local equilibrium of the curved film with vapour

$$
\Pi(h)+(\gamma / r)=-(\rho R T / \mu) \ln \left(p / p_{s}\right)
$$
one can obtain the following expression for $\mathrm{K} / \mathrm{K}_{1}$ [3] :

$$
\frac{K}{K_{1}}=1-\frac{\rho^{2} R T(2 \pi R T / \mu)^{1 / 2}}{4 \eta \mu r^{2}\left(p_{m}-p_{0}\right)} p_{0} \frac{h^{3}(p)}{p} d p
$$

The second term in Eq. (1) characterize the capillary pressure in the film, where $\gamma$ is the surface tension of the Ilquid. The wetting films of nonpoler decane are stable due to the action of long range dispersion forces. The $\Pi(h)$ isotherm correlating the thickness of a film with the diffoining pressure acting in it, has the form $\Pi=A / 6 \pi h^{3}$, where $\mathrm{A}=1.6 \times 10^{-2} \mathrm{~J}$, the Hamaker constant. Its value was calculated on the basis of the spectral data of quartz and decane [4] in accordance with the macroscopic theory of dispersion forces [5] : Using Eq. (1), we shall find the isotherm of polymolecular adsorption $h(p)$. Than after integrating Eq. (2) we obtain:

$$
\frac{K}{K_{1}}=1+\frac{A(2 R T / \pi \mu)^{1 / 2}}{24 \eta r^{2}\left(p_{m}-p_{0}\right)} \ln \left[\frac{\ln \left(p_{0} / p_{s}\right)-(\mu \gamma / \rho r R T)}{\ln \left(p_{m} / p_{s}\right)-(\mu \gamma / \rho r R T)}\right]
$$

The theoretical dependence $K / K_{1}(r / \lambda)$, represented in Fig. 1 by a continuous curve, was plotted with Eq. (3), using the bulk values of $\gamma, \rho_{\text {This }}$, and $\eta$. The experimental points are located close to that curve. This the properties of the bulk liquid, and that the equations of viscous flow apply to these.

In Fig. 2 are presented the results of similar experiments carried out on water [3]. As is known, not only dispersion forces, but also electrostatic and structural forces act in the films of polar water [2]. This complicate the theoretical determination of $h(p)$ isotherm. In this case the known experimental isotherm $h(p)$ [4] was used to make calculations with Eq. (2). The thickness of the films is small, and the experimental data tally with the theoretical curve 1 for the Knudsen flow of vapour, when a low relative pressure of vapaur is maintained in the chamber (points 1). Now, at higher values of $p_{0} / p_{g}=0.97-0.98$ the experimental points are located close to the curve 2, which was plotted by using Eg. (2). However, the theory can be in agreement with the experimental data (points 2 and 3 ) only when 4 times higher values of film viscosity $\eta$ as compared with bulk viscosity $\eta$ are adopted. Yet the viscosity of water can harly be increased so much. In accordance with the measurements done in fine pores $(r-2-3 \mathrm{~nm})$ of silica gels and porous glasses [2] the largest changes in the viscosity of water amount to 1.5 ? $0^{\circ}$ The detected inorease in the viscous resistance is probably associaled with the formation of water film of nonuniform thickneas on the surface of quartz. This may be attributed to the microheterogeneity of the quartz surface, containing not only hydrophilic silanol areas, but also siloxane ones that are less hydrophilic. As a result, the thicker films that are formed over the hydrophilic areas prove to be separated by thinner ones that give rise to a higher viscous resistance. The isotherms $h(p)$ of polymolecular water film on flat quartz surface, which are experimentelly obteined by the ellipsometric method, are characteriatic of only the mean thickness of the film.

As appears from Fig. 2, for water films the equations of viscous flow may be also formally used, but in this case including some effective values of either viscosity or thickness of the film. However, it is difficult to predict theoretically these effective values. To obtain more reliable 


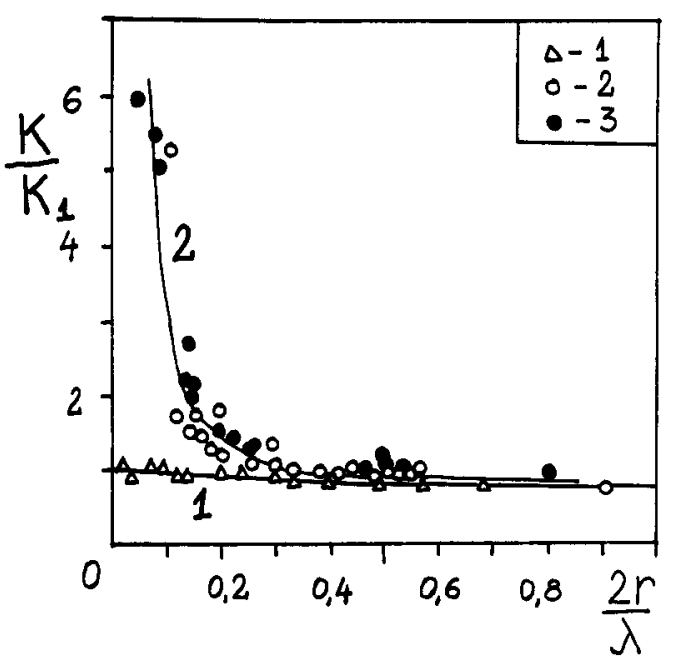

Fig. 2. The increase in the evaporation rate of water from fine capillaries owing to the flow of polymolecular adsorption flims of water. The relative pressure of vapour in the chamber: $p_{0} / p_{q}=0.75(1)$; 0.97 (2); and 0.98 (3)

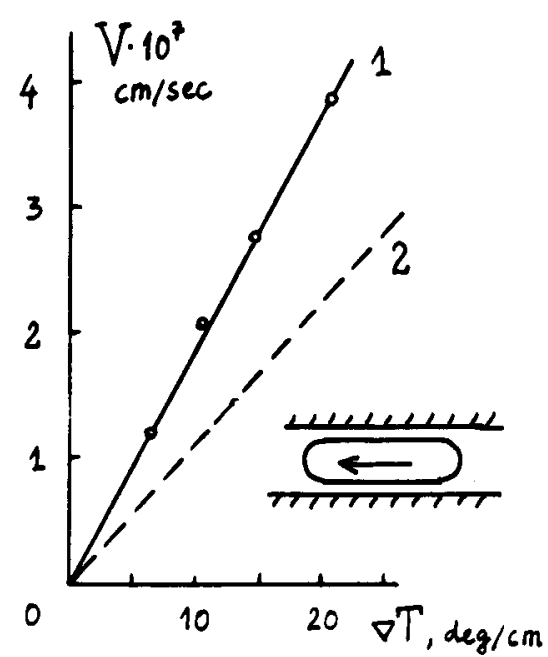

F18. 3. The Influence of the thermocapillary flow of dodecane films $(\mathrm{h}=2.5 \mathrm{~nm}$ ) on the rate of menisci displacement $V$ In a glass capillary at mean temperature of $+31^{\circ} \mathrm{C}$ 。

quantitative data on the polymolecular films of water requires carrying out the experiments on more uniform surfaces.

In the general case of heterogeneous aurfaces such a transport mechanism may be realized, when the viscous flow over the areas of thicker films is combined with the surface diffusion of water molecules on the "dry" areas separating the films from one another.

Thermocapillary film flow The second applied technique consisted in obcerving the film flow over the portion of the capillary located between the menisci (Fig. 3) [6]. The flow of the films occurred under the effect of the temperature gradient $\nabla \mathrm{T}$ which gave rise to the gradient of the aurface tension of the film. The velocity of the gas bubble shift $V$ is determined by the thermocapilary flow of the film $V_{f}$, and the diffusian of vapour $V_{d}: \quad V=V_{f}+V_{d}=\left[\left(h^{2} / r \eta\right)(\partial \gamma / \partial T)+(\mu D / \rho R T)(\partial p / \partial T)\right] \nabla T$,

where $D$ is the coefficient of diffusion of vapour through gas. The film flow rate was calculated from Eq. (4) as the difference between the directly measured velocity of bubble shift $V$, and the calculated value of $V_{f}$. In Fig. 3 are shown the results for low volatile dodecane in a glass capillary $r=0.73 \mu \mathrm{m}$. The experimental dependence $V(\nabla T)$ (curve 1) is linear in agreement with $\mathrm{Eq}$. (4). The dashed line 2 indicate the $V$ values calculated according to Eq. (4) using the known values of $D$ and $\mathrm{dp} / \mathrm{dT}$ for dodecane. The difference between the curves 1 and 2 gives the rate of the film flow, amounting up to about $40 \%$ of the total flow rate.

The obtalned values of $v_{f}$ tally well with those calculated by the aid of Eq. (4), using the bulk values of $\gamma$ (T) and $\eta$ for dodecane. The thickness of films was determined from the isotherms $\Pi m A / 6 \pi h^{3}=\gamma / r$, with the Hamaker constant $A=1.35 \times 10^{-2} \mathrm{~J}$ [4]. The applicability of the equations of viscous flow was corroborated by the series of experiments done on the capillaries having the radil from 0.4 to $17 \mu \mathrm{m}$, which corresponds to the film thicknesses from 2 to $10 \mathrm{~nm}$. The thermocapillary flow of water films is complicated by the hysteresis of the contact angle. In distinction to dodecane, water is able to wet quartz but partially. Between the retreating and advancing menisci arise a difference in the preseure $\Delta P=(2 \gamma / r)\left(\right.$ Cos $\left.\theta_{R}-\operatorname{Cos} \theta_{A}\right)$ that caused 
the counter flow in the film. However, in the case of relatively thick water films ( $h=5-10 \mathrm{~nm}$ ) the flow may also be described by the viscous flow equations.

Thermocrystallization film flow We have eliminated the influence of hysteresis, when examining the thermal flow of nonfreezing, polymolecular adsorbed water films between two ice mentsci in a capiliary. The flow of a liquid films is accompanied by the phase transitions taking place on the menisci. In this case the thermocrystallization film flow occurs, that is described by the following equation [7] :

$$
V_{f}=\left(2 \rho L \mathrm{~h} / 3 \eta r \rho_{i} T\right) \dot{\nabla} T
$$

where I is heat of fusion, and $\rho_{i}$ is the density of ice.

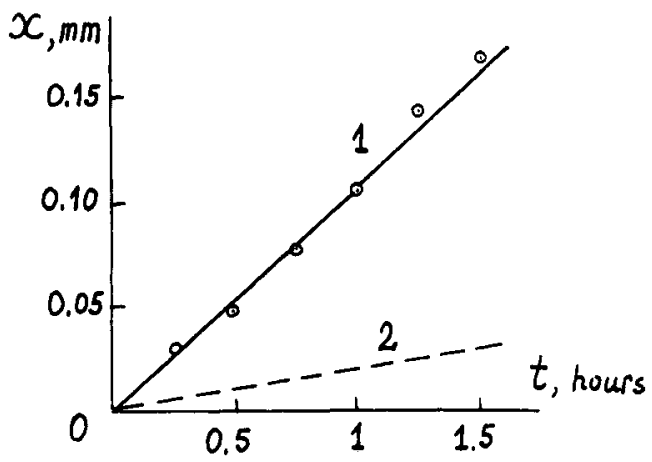

Fig. 4. Microscopilally measured ice menisci displacement $x$ versus time $t$ in quartz capillary ( $r$. $2.5 \mathrm{\mu m}$ ) at $-0.56^{\circ} \mathrm{C}$, and $\nabla \mathrm{T}$. 12.4 degrees/cm.

In Fig. 4 is shown one of the experimentally obtained dependences $x(t)$. As In FIg. 3, the dashed line 2 indicates the calculated contribution of the diffusion of vapour. A low pressure of vapour over lce, and the excess gas pressure in a bubble (increased up to 1.7 atm by sealing up the capillary in the nitrogen medium), enabled one to suppress the diffusion of vapour

[6] - On the basis of $x(t)$ dependences one can calculate the rate of displacement $\quad \mathrm{V}=\mathrm{v}_{f}+\mathrm{v}_{\mathrm{d}}=\mathrm{dx} / \mathrm{dt}$ and compare the results with theory. Measurements done in capillarles having the radif from 1 to $10 \mu \mathrm{m}$ within the temperature range of minus $6^{\circ} \mathrm{C}$ to minus $0.5^{\circ} \mathrm{C}$ have show that the flow of nonfreezing films from 1 to $8 \mathrm{~nm}$ thick obeys the $\mathrm{Eq}$ 。 (5).

Conclusion The films of nonpolar liquids having the thickness $h \geqslant 1 \mathrm{~nm}$ flow as viscous bulk liquids. This approach may also be applied to polymolecular water films; yet a possible nonuniformity in the thickness of thin polymolecular water films on microheterogeneous, only partlally wettable surfaces interferes with the quantitative comparison with theory.

\section{REFERENCES}

1. Z.M. Zorin, A.V。 Novikova and NoV. Churaev, In: Problems on the Physics of Formation and Phase Transformations, po42, Tula Polytech, Inst. USSR (1973).

2. B.V. Derjaguin, N.V. Churaev and V.M. Muller, Surface Forces, Plenum Press, New York (1987)。

3. Z.M. Zorin, A.V. Novikova, A.K. Petrov and N.V. Churaev, In: Surface Forces in Thin Films, and Stability of Colloids, p. 94, Nauka, Moscow (1974).

4. B.V. Derfaguin and NoV. Churaev, Wetting Films, Nauka, Moscow (1984).

5. I.E. Dzyaloshinsky, E.II. Lifshitz and L.P. Pitaevsky, Adv. Phys., 10, 165-187 (1961)。

6. B.V. Derjaguin, N.V. Churaev, O.A. Kiseleva and V.D. Sobolev, Langmulr, 3, 631-634 (1987).

7. B.V. Derjaguin, N.V. Churaev, V.D。 Sobolev and S.S. Barer, J. Colloid and Interface Sci., 84, 182-190 (1981). 